\begin{tabular}{|c|l|}
\hline Title & Light-stimulated root elongation in A rabidopsis thaliana \\
\hline Author(s) & Kurata, Tetsuya; Y amamoto, Kotaro T. \\
\hline Citation & Journal of Plant Physiology, 151(3), 346-351 \\
\hline Issue Date & 1997 \\
\hline Doc URL & http://hdl.handle.net/2115/44841 \\
\hline Type & article (author version) \\
\hline File Information & Kurata and Y amamoto, 1997.pdf \\
\hline
\end{tabular}

Instructions for use 


\section{Light-stimulated root elongation in Arabidopsis thaliana*}

\section{Tetsuya Kurata and Kotaro T. Yamamoto}

Division of Biological Sciences, Graduate School of Environmental Earth Science, Hokkaido University, Sapporo, 060 Japan

\section{Summary}

We investigated the effects of continuous irradiation either with white light or far-red light on elongation of roots of Arabidopsis thaliana. The white light used lacked most spectral components in the far-red region. The white-light irradiation of a whole seedling stimulated root growth; DCMU, an inhibitor of photosynthesis, completely inhibited this stimulation. Excised roots lost their responsiveness to light. Sugars produced during photosynthesis stimulated the elongation of roots in the dark. Root growth was also promoted by continuous exposure of a whole seedling to far-red light although chlorophyll did not accumulate at the same rate as under white light. The effect of far-red light was not inhibited by DCMU. In a phyA mutant, there was no stimulation of root growth under far-red light. These results show that root growth is primarily promoted by photosynthetic activity, and that phytochrome A stimulates root growth independently of photosynthesis. In a phyB mutant, there was no stimulation of root growth by either white, red or far-red light, suggesting that phytochrome B, either as Pr or Pfr, is necessary for the roots to respond 
to light.

Key words: Arabidopsis thaliana, elongation, photosynthesis, phytochrome, root.

Abbreviations: DCMU = 3-(3,4-dichlorophenyl)-1,1-dimethylurea; $\mathrm{Pr}, \mathrm{Pfr}=$ Red and far-red absorbing form of phytochrome, respectively.

*Dedicated to Professor Pill Soon Song on the occasion of his 60th birthday. 


\section{Introduction}

Plant development is regulated by various environmental factors. Light is one of the most important factors, and the effect is most obvious at the seedling stage. In dicotyledonous seedlings, light stimulates hook opening and cotyledon expansion and inhibits hypocotyl growth (Kendrick and Kronenberg, 1994). The inhibition of hypocotyl growth has been studied intensively and used for the isolation of photomorphogenic mutants (Koornneef et al., 1980).

The root is responsible for water and nutrient uptake from the soil and also responds to various stimuli, allowing plants to respond in the proper manner (Russell, 1977). Although responses of roots to light have been less well characterized than those of aerial organs, they have been investigated with respect to several physiological phenomena. Light sometimes promotes a positive gravitropic response in root (Lake and Slack, 1961) and, in the case of Arabidopsis, induces negative phototropism (Okada and Shimura, 1992; Liscum and Briggs, 1996); formation of lateral roots in pea is inhibited by continuous or daily-pulsed light (Torrey, 1952); light also decreases the effectiveness of IAA on the initiation of adventitious roots (Went and Thimam,1937); and elongation of root hairs in Arabidopsis is inhibited by light perceived by phytochrome B (Reed et al., 1993).

Effects of light on the elongation of roots have also been studied in both monocotyledonous and dicotyledonous plants (Mohr, 1961; Feldman, 1984). In all of the studies except one (Shen-Miller, 1974) light inhibited 
root elongation, irrespective of whether roots were intact or excised (Torrey, 1952; Björn et al, 1963). Irradiation with pulsed light was as inhibitory as with continuous light (Torrey, 1952); Schwarz and Schneider, 1985). Red light was most effective with maize roots (Schwarz and Schneider, 1985), while blue light was more effective with excised wheat roots (Björn et al., 1963). With rice seedlings, blue light inhibited root growth through suppression of both cell elongation and division, but red or far-red light inhibited only cell elongation (Ohno and Fujiwara, 1967). The use of the photosynthetic inhibitor, DCMU, showed that photosynthetic activity was not required for the photoinhibition (Ohno and Fujiwara, 1967).

Arabidopsis provides a very powerful experimental system for the identification of photoreceptors of photomorphogenic responses, since an array of photoreceptor mutants has been established in the last 15 years (Koornneef et a1., 1980; Kendrick and Kronenberg, 1994; Quail et al., 1994). While raising Arabidopsis seedlings vertically on solidified medium in light and dark conditions, we have noticed that the root is much longer in the light than in the dark, which contradicts most of the reports described in the literature. Thus, we started to characterize the light-stimulated elongation of Arabidopsis roots. In the present study, taking advantage of an inhibitor of photosynthesis and a number of photoreceptor mutants, we found that the photostimulation of root growth was primarily a consequence of independent effects of photosynthesis and phytochrome A. Phytochrome B was also a prerequisite for the root to respond to light.

\section{Materials and Methods}


Plant materials and growth conditions

Arabidopsis thaliana (L.) Heynh. Columbia-0 ecotype was used in this study. Landsberg erecta ecotype was also used for experiments using the phy $B-1$ (Quail et al., 1994) and the phyA-302 mutants which are in a Landsberg erecta background. The phyA-302 mutant was isolated in our laboratory as a long hypocotyl mutant under continuous far-red light from seeds mutagenized by fast neutrons (Lehle Seeds, Round Rock, TX, USA); it was allelic to the phyA-102 mutant (Parks and Quail, 1993; Dehesh et al. 1993; Quail et al., 1994).

Arabidopsis seeds were surface sterilized with $70 \%$ ethanol for 5 min, and next with $1.5 \%$ sodium hypochlorite and $0.02 \%$ Triton X-100 for 15 min. After washing five times with sterile $\mathrm{H}_{2} \mathrm{O}$, seeds were sown onto a 1/2 MS medium plate containing $1 \%$ Gellan Gum, Gamborg $\mathrm{B}_{5}$ vitamin, and $1 \%$ sucrose. The plates were incubated at $4^{\circ} \mathrm{C}$ for 2 to $4 \mathrm{~d}$, and then illuminated with red light for $6 \mathrm{~h}$ at $22^{\circ} \mathrm{C}$ to induce germination. The plates were then placed in a vertical position at 22 to $24^{\circ} \mathrm{C}$, so that the seedlings were grown along the surface of the solidified medium. For inhibitor experiments, DCMU (Sigma, St. Louis, MO, USA) solutions of various concentrations in ethanol were added to the medium at a 1,000-fold dilution. Therefore, the final concentration of ethanol in the medium was always kept $0.1 \%$.

\section{Light source}

White light $\left(43 \mathrm{~W} \mathrm{~m}^{-2}\right)$ was obtained from five $40 \mathrm{~W}$ white fluorescent light 
bulbs (FL40SS W/37, Sanyo, Tokyo, Japan). Broad-band red light (3.7 W $\mathrm{m}^{-2}$ ) was obtained from five $20 \mathrm{~W}$ white fluorescent light bulbs (FL20SS W/18, Matsushita, Osaka, Japan) filtered through a red acrylic filter (No. 102, Mitsubishi Rayon, Tokyo, Japan). Broad band far-red light (6.3 W

$\mathrm{m}^{-2}$ ) was obtained from three far-red-enriched fluorescent bulbs (FL20SS FR-74, Toshiba, Tokyo, Japan) filtered through a black acrylic filter (IR-70, Mitsubishi Rayon). Fluence rates were measured with an optometer (Model 370, Graseby Optronis, Orland, FL, USA).

\section{Measurement of length}

Root and hypocotyl lengths were measured from images of a seedling taken by a CCD camera (XC77, SONY, Tokyo, Japan) with a TV zoom lens (J6 x 11-II, Canon, Tokyo, Japan), and captured to a desktop computer (PC9801 BA2, NEC, Tokyo, Japan). The image was measured using image analyzing software (RIPP-2 ver.1.05, Rise, Sendai, Japan).

\section{Measurement of oxygen evolution}

The concentration of dissolved oxygen was measured at room temperature with an oxygen analyzer (Model 778, Beckman, Fullerton, CA, USA) connected to an analogue recorder (QPD54, Hitachi, Tokyo, Japan). Ten 6-day-old light-grown Arabidopsis seedlings were used for each measurement. An aerial part of the seedling was cut into sections of approximately $1 \mathrm{~mm}$ square. The sections were subjected to vacuum for 1 min twice in $0.5 \mathrm{mmol} \mathrm{L}^{-1} \mathrm{CaSO}_{4}$ and $50 \mathrm{mmol} \mathrm{L}^{-1} \mathrm{HEPES}$ (N-[2-hydroxyethyl]piperazine-N'- [2-ethanesulfonic acid]) (pH 7.2), using a 
vacuum pump (4VP-C2, Hitachi). A cuvette equipped with a magnetic stirrer was filled with the buffer and preilluminated for 6 min with white light $\left(220 \mathrm{~W} \mathrm{~m}^{-2}\right)$ provided from an illuminator (FLH-50, Shimadzu, Kyoto, Japan). The plant sample was put in the cuvette and incubated for 6 min under the illumination. $\mathrm{NaHCO}_{3}$ was then added into the buffer at a final concentration of $20 \mathrm{mmol} \mathrm{L}^{-1}$, and the sample was incubated for another 6 min with constant stirring. Finally, DCMU dissolved in ethanol from 0.1 mmol L-1 to1 $\mathrm{mmol} \mathrm{L}^{-1}$ was added at a 1,000-fold dilution. The final concentration of ethanol in the reaction buffer was always $0.1 \%$. The relative oxygen evolution was calculated as the ratio of the rate of oxygen evolution after the addition of DCMU to that before its addition.

\section{Measurement of chlorophyll content}

One hundred seedlings grown for $6 \mathrm{~d}$ were soaked in $\mathrm{N}, \mathrm{N}^{-}$ dimethylformamide at a ratio of $1: 12.5(\mathrm{w} / \mathrm{v})$ for $1 \mathrm{~d}$ at $1^{\circ} \mathrm{C}$. The absorption of the solution was measured with a spectrophotometer (DU-65, Beckman), and the amount of total chlorophyll was estimated according to Moran (1982).

\section{Results}

\section{Effects of continuous light on root elongation}

After synchronous germination, induced by chilling and red-light irradiation, seedlings of Arabidopsis Columbia-0 ecotype were incubated under continuous white light or in the dark. Since seedlings were grown 
along the surface of vertically-oriented, solidified Gellan-Gum plate, whole seedlings including root were exposed to the light (Fig.1). The rate of root

elongation was similar in both conditions until the third day; in the light it then increased significantly, while in the dark it decreased slightly.

We then tested the fluence rate response for the light-induced stimulation of root growth (Fig.2). Significant stimulation of root elongation occurred at a fluence rate of $1.1 \mathrm{~W} \mathrm{~m}^{-2}$ and even greater stimulation was observed at $14 \mathrm{~W} \mathrm{~m}^{-2}$. The dose-response curve for the inhibition of hypocotyl growth was very similar.

\section{Effects of continuous light on elongation of isolated roots}

We next investigated the effects of light on the growth of roots that had been separated from the hypocotyl and the cotyledon by excision. In the dark, isolated roots grew as well as intact roots, but elongation of excised roots was not stimulated by continuous white light (Fig. 3), suggesting that substances transported from aerial parts of the seedling are essential for the light-stimulated elongation of roots.

\section{Effects of DCMU on light-stimulated elongation of roots}

The involvement of photosynthesis in the light-stimulated growth of roots was investigated using DCMU, an effective inhibitor of electron transport from photosystem II to I (Fig. 4). Arabidopsis seedlings were grown on DCMU-supplemented medium for $6 \mathrm{~d}$ in continuous white light or in darkness. On media without DCMU, roots were $2-3$ times longer in the light than in darkness. In the dark, root length was unaffected by DCMU, 
while in the light, root length was decreased by DCMU in a dose-dependent manner. At $0.4 \mu \mathrm{mol} \mathrm{L}-1$ DCMU and higher, there was no significant increase in root length. To confirm the inhibitory effect of DCMU on photosynthesis in the light, oxygen evolution of the light-grown seedlings was determined. DCMU inhibited oxygen evolution in a dose-dependent manner and was completely inhibitory at $1 \mu \mathrm{mol} \mathrm{L}^{-1}$ (Fig.4). Even in the absence of DCMU, oxygen evolution was decreased by $9 \%$, probably because of the presence of ethanol, used as a solvent for DCMU. The light-stimulated elongation of the roots was inhibited in a parallel manner to the decrease in oxygen evolution.

\section{Effects of sugar species on root elongation in the dark}

Since sugars are major products of photosynthesis, the effect of supplementing the basal medium with various sugars on the growth of roots after $6 \mathrm{~d}$ was determined (Fig. 5). In all of the experiments shown above, the culture medium contained $1 \%$ sucrose $\left(0.027 \mathrm{~mol} \mathrm{~L}^{-1}\right)$. The addition of sucrose stimulated root growth in the dark, though it was not as effective as the light treatment, and adding sucrose did not affect root growth in the light. Fructose and glucose also stimulated root growth in the dark. Sorbitol and mannose were both ineffective. These results indicate that sugar species that are effectively produced during photosynthesis stimulate root elongation in the dark.

\section{Effects of light on root elongation of phyA and phyB mutants}

Since the white light used in the present study was obtained from normal 
fluorescent tubes (day-light type), it contained only a small amount of far-red light ( $2.4 \%$ on an energy base). When the effect of far-red light on root growth was determined (Table l), it was found that continuous irradiation with far-red light induced root growth. The effect was not inhibited by $1 \mu \mathrm{mol} \mathrm{L} \mathrm{L}^{-1} \mathrm{DCMU}$, and excised roots did not respond to far-red light (data not shown).

The above results suggested that phytochrome was involved in the response. Therefore we investigated root growth of the phytochrome-deficient mutants, phyA-302 and phyB-1, under continuous red, far-red and white lights. The chlorophyll content of the seedlings was also determined, as a measure of their capacity for photosynthesis (Fig. 6). The Landsberg erecta ecotype was used as wild type in this experiment, since the mutants were isolated in this background. Although root growth in this ecotype was less responsive to white light than in the Columbia-0 ecotype (Fig. 6 vs. 2), irradiations with either red or white light induced both promotion of root growth and accumulation of chlorophyll. Far-red light irradiation, in contrast, stimulated root growth, but did not result in chlorophyll accumulation. Roots of the two mutants displayed different growth responses to light. Irradiation with far-red light failed to stimulate root growth in the phyA mutant, while red and white light increased root growth and chlorophyll content to an extent similar to that in the wild type. In the case of the phyB mutant, none of the light treatments stimulated root growth (statistically tested by $t$ test with $P<0.05$ ) although white light induced a considerable accumulation of chlorophyll. These results indicate that both phytochrome A and B can influence root growth, although their 
modes of action seem different.

\section{Discussion}

We have observed that continuous irradiation of a whole seedling with white light stimulates root elongation in Arabidopsis. This response occurred at fluence rates that inhibited hypocotyl growth (Fig. 2). Root elongation was effectively inhibited by DCMU, an inhibitor of photosynthesis, indicating that photosynthesis was involved in the stimulation. The addition of sugars to the medium promoted root growth in the dark, but only sugars produced during photosynthesis (sucrose, glucose, and fructose) were effective (Fig. 5). Roots lost their responsiveness to light when excised from the cotyledon and hypocotyl (Fig. 3). These results are also consistent with the conclusion that photosynthesis is the primary driving force for the light-induced root growth. A recent study reveals that sugars often affect physiological processes as a signal molecule (Cheng et al., 1992; Jang and Sheen, 1994). However, it is currently not known whether photosynthesis stimulates root growth through an increased energy supply or whether some product is acting as a signal.

It was also found that continuous irradiation with far-red light promoted root growth (Table 1 and Fig. 6). Seedlings grown under far-red light contained only 1/100 as much chlorophyll as those grown under continuous white light, indicating that development of the photosynthetic machinery was considerably suppressed under far-red light. These results 
indicate that a photoreceptor(s) other than photosynthetic pigments are involved in the far-red promotion of root growth. The phyA mutant used specifically lacked a response to far-red light (Fig. 6), which, together with the effects of far-red light observed in the wild type, clearly indicates that phytochrome A is responsible for the far-red-light induced promotion of root growth. In contrast to the phy $A$ mutant, the $p h y B$ mutant did not show any response to red, far-red or white light.

Growth of the cotyledon and hypocotyl is regulated by the light environment. It is well known that the inhibition of hypocotyl growth by blue, red and far-red light is mediated by a blue-light photoreceptor (HY4 or CRY1), phytochrome B and phytochrome A, respectively (Kendrick and Kronenberg, 1994; McNellis and Deng, 1995). For stimulation of cotyledon expansion by red light, both phytochrome A and B are required while, under far-red light, only phytochrome A is responsible (Reed et al., 1994). The cotyledon expansion elicited by phytochrome B is cell autonomous (Neff and Van Volkenburgh, 1994), while that induced by blue light is only observed in intact plants (Blum et al., 1994). We observed that cotyledon expansion induced by continuous white light was not blocked by DCMU (data not shown). These results suggest that photocontrol of growth is different in the roots and in the aerial parts of a seedling.

Roots develop post-embryonically from the meristematic region through cell division and expansion (Dolan et al., 1993). In contrast, cotyledon and hypocotyl develop through embryogenesis and expand without cell division as post-embryonic development in Arabidopsis (Tsukaya et al., 1994; Desnos et al., 1996). Light-stimulated root 
elongation may be dependent on an increase in cell division activity, which may require more photosynthetic products.

Neither red, nor far-red, nor white light promoted root growth in the phy $B$ mutant, despite a considerable accumulation of chlorophyll under white light. These results suggest that the source-sink relationship between cotyledons and roots may be disrupted in the $p h y B$ mutant in an unknown manner. The phyB-I mutant has a nonsense mutation that blocks synthesis of the phytochrome B apoprotein (Reed et al., 1993). Thus, the results also suggest that the Pr form of phytochrome B as well as the Pfr form is physiologically active in this response, which has been proposed recently (Smith, 1995). Robson and Smith (1996) recently investigated negative gravitropism of hypocotyl of a few alleles of the phyB mutant in the dark and suggested that the phyB-1 mutant, which was used in this study, contains a second mutation besides a mutation in $P H Y B$. If this is the case, the unresponsiveness of root elongation to light observed in the phy $B-1$ mutant may be ascribed to the second mutation.

In contrast to our observations, Reed et al. (1993) reported no differences in root length of Arabidopsis ecotype Landsberg erecta grown in the light or in the dark. This inconsistency may be due to differences in the growth conditions. We always cultured the seedlings in a medium containing 1\% sucrose, but Reed et al. (1993) used a medium containing $2 \%$ sucrose. Since sucrose stimulates root growth in the dark (Fig. 5), the presence of a higher concentration of sucrose might make the photostimulation of root growth less apparent. In our study, light was supplied continuously; Reed et al. (1993) cultured seedlings under a $16 \mathrm{~h}$ 
light/8 h dark photoperiod, which may not have been enough to stimulate root growth.

Although we demonstrated that root growth of Arabidopsis is promoted by light, most published results reported the opposite. We therefore checked root growth of several plant species. Growth of roots was inhibited by continuous white light in seedlings of mung bean, maize, and radish; no significant effects were observed in cabbage, lettuce, and tobacco (data not shown). It is concluded that light-induced promotion of root growth is not a universal photomorphogenic response.

In conclusion, the present study shows that root growth is stimulated by continuous light in Arabidopsis. The effect is due to photosynthetic activity and to the action of phytochrome A. Phytochrome B also has a significant role in this phenomenon through an unknown action.

\section{Acknowledgements}

We thank Marten Koornneef for providing us with seeds of phyB-1. We also thank Yasuhiro Takada for making an oxygen analyzer available to us. This work was supported in part by a Grant-in-Aid from the Japanese Ministry of Education, Science and Culture (No. 05680631 and 05276102) to K.T.Y. 


\section{References}

Björn, L.O., Y. Suzuki, and J. Nilsson: Influence of wavelength on the light response of excised wheat roots. Physiol. Plant. 16, 132-141 (1963).

Blum, D.E., M.M. Neff, and E. Van Volkenburgh: Light stimulated cotyledon expansion in the blu3 and hy4 mutant of Arabidopsis thaliana. Plant Physiol. 105, 1433-1436 (1994).

Cheng,C.-L., G.N. Acedo, M. Cristinsin, and M.A. Conkling: Sucrose mimics the light induction of Arabidopsis nitrate reductase gene transcription. Proc. Natl. Acad. Sci. USA 89, 1861-1864 (1992).

Dehesh, K., C. Franci, B.M. Parks, K.A. Seeley, T.W. Short, J.M. Tepperman, and P.H. Quail: Arabidopsis HY8 locus encodes phytochrome A. Plant Cell 5, 1081-1088 (1993).

Desnos, T., V. Orvovic, C. Bellini, J. Kronenberger, M. Caboche, J. Traas, and H. Höfte: Procuste1 mutants identify two distinct genetic pathways controlling hypocotyl cell elongation, respectively in dark- and light-grown Arabidopsis seedlings. Development 122, 683-693 (1996).

Dolan, L., K. Janmaat, V. Willemsen, P. Linstead, S. Poethig, K. Roberts, and B. Scheres: Cellular organization of the Arabidopsis thaliana root. Development 119, 71-84 (1993).

Feldman, L.J.: Regulation of root development. Annu. Rev. Plant Physiol. 35, 223-242 (1984).

Jang, J.-C., and J. Sheen: Sugar sensing in higher plants. Plant Cell 6, 1665-1679 (1994). 
Kendrick, R.E. and G.H.M. Kronenberg eds.: Photomorphogenesis in Plants, 2nd ed. Kluwer, Dordrecht (1994).

Koornneef, M., E. Rolff, and C.J.P. Spruit: Genetic control of light-inhibited hypocotyl elongation in Arabidopsis thaliana (L.) Heynh. Z. Pflanzenphysiol. 100, 147-160(1980).

Lake, J. V. and G. Slack: Dependence on light of geotropism in plant roots. Nature 191, 300-302 (1961).

Liscum, E. and W.R. Briggs: Mutations of Arabidopsis in potential transduction and response components of the phototropic signaling pathway. Plant Physiol. 112, 291-296 (1996).

McNellis, T.W. and X.-W. Deng: Light control of seedling morphogenetic pattern. Plant Cell 7, 1749-1761 (1995).

Mohr, H.: Wirkungen kurzwelligen Lichtes. In: Ruhland, W. (ed.): Handbuch der Pflanzenphysiologie, Vol. 16, 439-531. Springer, Berlin, 1961.

Moran, R.: Formulae for determination of chlorophyllous pigments extracted with N,N-dimethylformamide. Plant Physiol. 69, 1376-1381 (1982).

Neff, M.M. and E. Van Volkenburgh: Light-stimulated cotyledon expansion in Arabidopsis seedlings. Plant Physiol. 104, 1027-1032 (1994).

Ohno, Y. and A. Fujiwara: Photoinhibition of elongation growth of roots in rice seedlings. Plant Cell Physiol. 8, 141-150 (1967).

Okada, K. and Y. Shimura: Mutational analysis of root gravitropism and phototropism of Arabidopsis thaliana seedlings. Aust. J. Plant Physiol. 19, 439-448 (1992). 
Parks, B.M. and P.H. Quail: hy8, a new class of Arabidopsis mutants deficient in functional phytochrome A. Plant Cel1 5, 39-48 (1993).

Quail, P. H., W.R. Briggs, J. Chory, R.P. Hangarter, N.P. Harberd, R.E. Kendrick, M. Koornneef, B. Parks, R.A. Sharrock, E. Schäfer, W.F. Thompson, and G.C. Whitelam: Spotlight on phytochrome nomenclature. Plant Cell 6, 468-471 (1994).

Reed, J.W., A. Nagatani, T.D. Elich, M. Fagan, and J. Chory: Phytochrome A and phytochrome B have overlapping but distinct functions in Arabidopsis development. Plant Physiol. 104 1139-1149 (1994).

Reed, J.W., P. Nagpal, D.S. Poole, M. Furuya, and J. Chory: Mutations in the gene for the red/far-red light receptor phytochrome B alter cell elongation and physiological responses throughout Arabidopsis development. Plant Cell 5, 147-157 (1993).

Robson, P.R. and H. Smith: Genetic and transgenic evidence that phytochromes A and B act to modulate the gravitropic orientation of Arabidopsis thaliana hypocotyls. Plant Physiol. 110, 211-216 (1996).

Russell, R.S.: Plant root systems. McGraw-Hill, London (1977).

Schwarz, H. and H.A.W. Schneider: Light-mediated growth inhibition of maize roots. J. Plant Physiol. 119, 281-284 (1985).

Shen-Millar, J.: Spectral sensitivity of corn־root geotropism. In: Plant Growth Substances, Hirokawa, Tokyo, 1095-1103 (1973).

Smith, H.: Physiological and ecological function within the phytochrome family. Annu. Rev. Plant Physiol. Plant Mol. Biol. 46, 289-315 (1995). Torrey, J.G.: Effect of light on elongation and branching in pea roots. Plant Physiol. 27, 591-602 (1952). 
Tsukaya, H., T. Tsuge, and H. Uchimiya: The cotyledon: a superior system for studies of leaf development. Planta 195, 309-312 (1994).

Went, F.W. and K.V. Thimann: Phytohormones. Macmillan, New York (1937). 
Table 1: Effect of continuous irradiation with far-red light on root growth in the presence or absence of $1 \mu \mathrm{mol} \mathrm{L}-1$ DCMU.

Light treatment DCMU

Dark

Dark

Far-red light

Far-red light

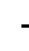

$+$

-
Root length $(\mathrm{mm})$

$16.5 \pm 2.3^{\mathrm{a}}$

$14.7 \pm 1.9$

$22.1 \pm 2.5$

$22.5 \pm 3.2$

After induction of germination, seedlings were grown at $22^{\circ} \mathrm{C}$ for $5 \mathrm{~d}$ under continuous far-red light $\left(6.3 \mathrm{~W} \mathrm{~m}^{-2}\right)$ or in the dark. For more details, see the legend to Fig. 1.

a The mean of at least 10 seedlings $\pm \mathrm{SD}$. 


\section{Figure legends}

Fig. 1: Growth of roots under continuous white light (open circles) or in the dark (closed circle). After seeds of Arabidopsis thaliana Columbia-0 ecotype were sown on $1 \%$ Gellan-Gum plate and chilled at $4^{\circ} \mathrm{C}$ for $4 \mathrm{~d}$, they were illuminated under red light at $22^{\circ} \mathrm{C}$ for $6 \mathrm{~h}$ to induce germination. Seedlings were then grown on vertically־oriented plates under continuous white light $\left(43 \mathrm{~W} \mathrm{~m}^{-2}\right)$ or in the dark at $22^{\circ} \mathrm{C}$ for the indicated number of days. The mean root length of at least 10 seedlings is shown $\pm \mathrm{SD}$.

Fig. 2: Fluence rate responses for growth of root (circles) and hypocotyl (squares). After induction of germination, seedlings were grown under continuous white light of various fluence rates at $22^{\circ} \mathrm{C}$ for $6 \mathrm{~d}$. Root and hypocotyl lengths are expressed relative to their length in the dark. The mean root length of at least 18 seedlings is shown \pm SD. For more details, see the legend to Fig. 1.

Fig. 3: Growth of excised roots under continuous white light (open bar) or in the dark (closed bar). After induction of germination, seedlings were incubated in the dark at $22^{\circ} \mathrm{C}$ for $2 \mathrm{~d}$ on $1 \%$ Gellan Gum plate and roots were then excised. The excised roots were cultured on the plate for additional $5 \mathrm{~d}$. The mean length of at least 11 roots is shown $\pm \mathrm{SD}$. For more details, see the legend to Fig. 1. 
Fig. 4: Effect of DCMU on root length (circles) and oxygen evolution (squares) under continuous white light. Seeds were sown on 1\% Gellan-Gum plate supplemented with the indicated concentration of DCMU. After induction of germination, seedlings were grown under continuous white light (open circles) or in the dark (closed circles) at $22^{\circ} \mathrm{C}$ for $6 \mathrm{~d}$. The mean root length of at least 18 seedlings is shown \pm SD. The rate of oxygen evolution was measured in the seedlings grown under white light. Relative oxygen evolution was calculated as a ratio of the rate of oxygen evolution after the addition of DCMU to that before its addition. For more details, see the legend to Fig. 1.

Fig. 5: Effect of sugars on growth of roots under continuous white light (open bar) or in the dark (closed bar). Seeds were sown on 1\% Gellan Gum plate supplemented with the indicated sugar at a final concentration of $0.027 \mathrm{~mol} \mathrm{~L}^{-1}$. After induction of germination, seedlings were grown at $22^{\circ} \mathrm{C}$ for $6 \mathrm{~d}$ in each light condition. The mean root length of at least 10 seedlings is shown \pm SD. For more details, see the legend to Fig. 1.

Fig. 6: Effects of continuous irradiation with white, red and far-red light on root length and chlorophyll content of seedlings of wild type (Landsberg erecta ecotype) (circles), the phy $A-302$ (triangles), and the phy $B-1$ mutants (squares). Fluence rates of the white, red and far-red light were 43, 3.7, 6.3 $\mathrm{W} \mathrm{m} \mathrm{m}^{-2}$, respectively. The mean root length of at least 12 seedlings is shown \pm SD. For more details, see the legend to Fig. 1. 


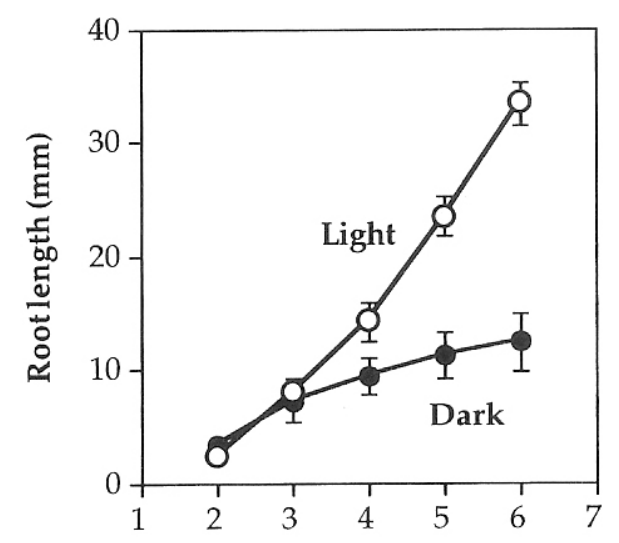

Days after transferring to light or dark

Fig. 1

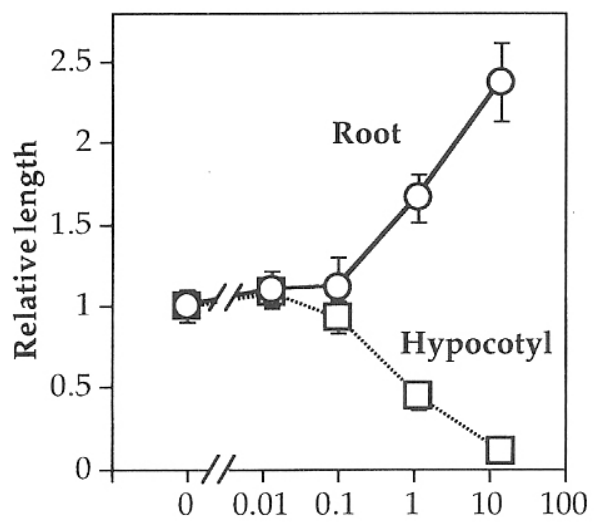

Fluence rate $\left(\mathrm{W} \cdot \mathrm{m}^{-2}\right)$

Fig. 2

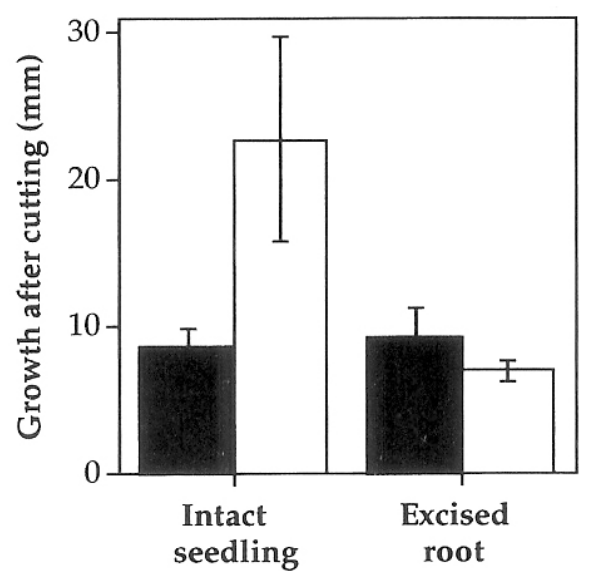

Fig. 3 


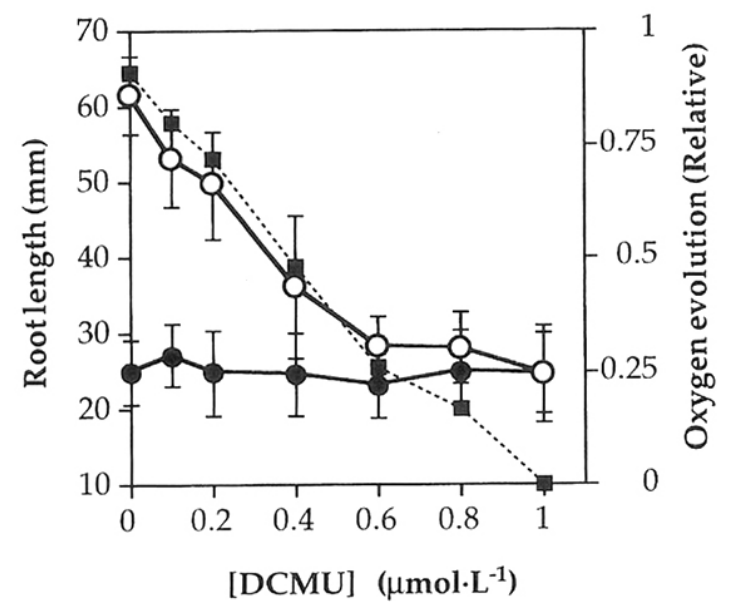

Fig. 4

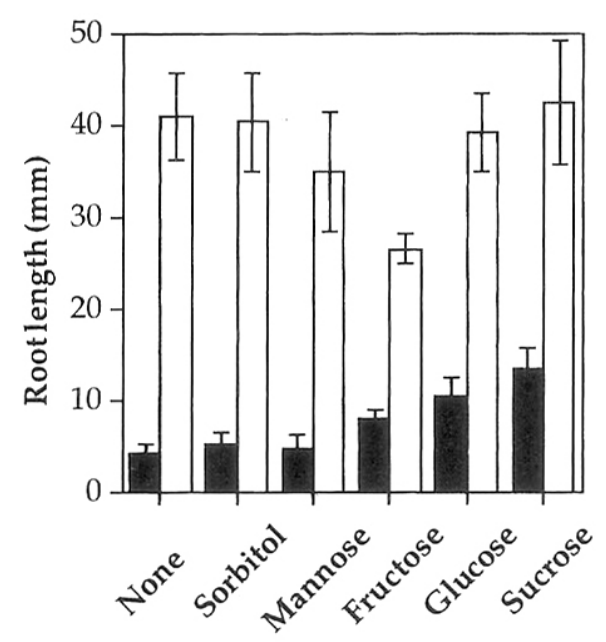

Fig. 5

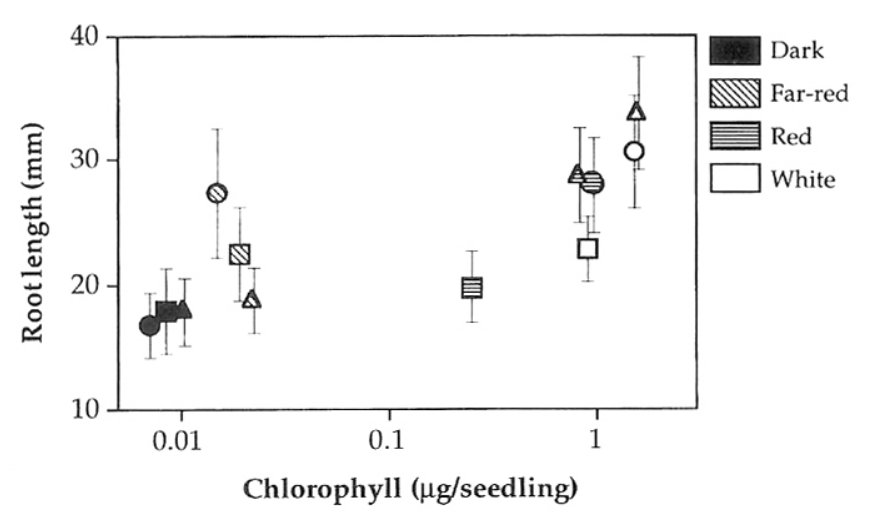

Fig. 6 